\title{
La influencia del nivel de estudios de padres de familia en el rendimiento de estudiantes de la educación media superior
}

Irvin Rodolfo Tapia Bernabé

irtb.tapia@gmail.com

Colegio de Educación Profesional Técnica del Estado de México

Recibido:11-noviembre-2019

Aceptado:30-enero-2020

\section{Resumen}

En las últimas décadas, la investigación educativa se ha dado a la tarea de conocer la relación de los factores socioculturales, socioeconómicos y de la propia escuela en el desempeño académico y en el logro de los aprendizajes de los estudiantes. El presente estudio, aporta evidencia empírica para dar respuesta al cuestionamiento ¿Influye el nivel de estudios de los padres de familia en el rendimiento escolar de los estudiantes? Bajo los supuestos de las teorías relacionadas a la influencia de los padres en el desarrollo de los hijos, se analizaron los resultados de una prueba estandarizada aplicada a una muestra de 10,110 estudiantes de sexto semestre de la educación media superior y un cuestionario de contexto. Para ello, se empleó el modelo estadístico no paramétrico de Chi Cuadrada para conocer la intensidad de las variables del tipo categórico. Los resultados arrojaron que los estudiantes con padres de familia con niveles de estudios de nivel medio superior y superior, obtienen mejores desempeños en pruebas estandarizadas del logro del aprendizaje.

Palabras clave: Escolaridad, Rendimiento Académico, Evaluación estandarizada, Logro educativo.

\section{The influence of the level of studies of parents on the performance of students in upper secondary education}

\begin{abstract}
In recent decades, educational research has been given the task of knowing the relationship of socio-cultural, socioeconomic factors and the school itself in academic performance and in the achievement of student learning. The present study provides empirical evidence to answer the question Does the level of studies of parents influence the students' school performance?


Under the assumptions of theories related to the influence of parents on the development of children, the results of a standardized test applied to a sample of 10110 sixth-semester students of upper secondary education and a context questionnaire were analyzed. To do this, the non-parametric Chi square statistical model was used to know the intensity of the categorical type variables. The results showed that students with parents with higher and middle school levels obtain better performances in standardized tests of achievement of learning.

Keywords: Schooling, Academic Performance, Standardized Evaluation, Educational Achievement.

\section{Introducción}

Actualmente, las pruebas estandarizadas del logro de los aprendizajes representan por su objetividad, confiabilidad y validez de sus instrumentos, uno de los mejores métodos para evaluar los sistemas educativos en el mundo. Al respecto, los resultados obtenidos en los últimos años de medición en México han demostrado serias deficiencias en el aprendizaje de las matemáticas y lenguaje y comunicación en jóvenes que culminan su trayecto formativo de la educación media superior. De acuerdo con (SEP, 2017) el dominio de este conjunto de aprendizajes constituye una herramienta fundamental para el desarrollo de nuevos aprendizajes en la educación superior o bien para insertarse de manera favorable al mundo productivo.

El campo de la investigación sobre los factores que inciden en el desarrollo de los aprendizajes ha demostrado que los resultados en el logro educativo no dependen en su totalidad de las capacidades de los estudiantes, del entorno familiar o de las características de la escuela. Si no que son resultado de la relación de diversas variables que giran en torno al estudiante y que impactan de forma negativa o positiva, según las características de los alumnos (INEE, 2015). Algunas de estas variables son las características sociodemográficas de los estudiantes, escolaridad del padre y la madre, las expectativas académicas, el trabajo, repetición escolar entre otras.

En este contexto, es importante el uso de modelos analíticos que permitan vincular los resultados de pruebas estandarizadas con diversas variables de tipo social, económico y 
educativo-institucional, con el fin de conocer los factores que inciden en el logro de los aprendizajes de los sistemas educativos. Por ello, el presente trabajo se encuentra centrado en conocer la influencia del nivel de estudios de los padres de familia en el desempeño de estudiantes de la educación profesional técnica, quien en el año 2017 ocupó la última posición en el ranking de subsistemas de la educación media superior en el país, según el informe de resultados de la Evaluación para el Logro de los Aprendizajes (INEE, 2017).

Teniendo presente que los resultados de pruebas estandarizadas aportan información valiosa para emprender programas de mejora encaminados a la mejora de la calidad educativa y para identificar los principales factores que inciden en los aprendizajes, se aprovecharon los resultados de la Evaluación Institucional del Núcleo de Formación Básica (EINFB) del Conalep Estado de México, para dar respuesta a la pregunta ¿Influye el nivel de estudios de los padres de familia en el rendimiento escolar de los estudiantes? Para ello, se empleó el método estadístico no paramétrico de Chi Cuadrado de Pearson, el cual es empleado para conocer la intensidad de asociación de dos variables categóricas. En este sentido, es importante comentar que la EINFB es una prueba estandarizada la cual ha demostrado su validez y confiabilidad en la evaluación de estudiantes de sexto semestre de sus 39 planteles, para medir la apropiación de los conocimientos del marco curricular común del bachillerato en sus cinco campos disciplinares.

\section{Marco teórico}

\section{El rendimiento escolar}

De acuerdo con (Morales, Morales, \& Holguín, 2016) el término de rendimiento escolar tiene su origen en el modelo económico industrial, el cual se caracterizó por introducir dimensiones objetivas y escalas para la medición de la eficiencia de productividad y calidad de los trabajadores, procesos, servicios, etc. En el ámbito educativo el rendimiento abarca una dimensión orientada a la medición de resultados y eficiencia, se aplica a alumnos, maestros, escuelas, entre otros. En la actualidad, se le ha definido al rendimiento escolar como el nivel de conocimientos demostrados en un área o materia comparado con la edad y nivel académico (Jiménez, 2000), aunque para (Martínez-Otero, 2007) es el producto que da el alumnado en los centros de enseñanza y que habitualmente se expresa a través de las calificaciones escolares. En ambos casos, el rendimiento escolar o académico refleja el grado 
en el que los estudiantes aprovechan los servicios educativos a partir del establecimiento de ciertos parámetros de medición.

\section{Factores que intervienen en el rendimiento escolar}

La investigación sobre los modelos causales en el rendimiento escolar y logro académico tiene sus orígenes en los estudios elaborados por (Coleman, y otros, 1966) donde se contemplaron cerca de 400 variables que influyen el logro educativo. Para (Page, 1990) atribuye en su modelo causal, determinantes del tipo contextual y personal del estudiante. En este modelo las variables contextuales contemplan aspectos sociofamiliares y variables escolares, y en las variables personales integra la inteligencia y aptitudes, estilos cognitivos, sexo y personalidad. En (Garbanzo, 2007) la dimensión de los factores asociados al rendimiento escolar en estudiantes de educación superior, tienen su relación con determinantes personales, sociales e institucionales. En la actualidad, el modelo propuesto (Backhoff, Monroy, \& Tanamachi, 2005) Marco de referencia para desarrollar instrumentos de contexto, dimensionan las variables según las características personales y socioculturales; infraestructura de la escuela y apoyos pedagógicos de los estudiantes; actividades que se relacionan con el proceso de enseñanza-aprendizaje; clima social y escolar en el que se desarrollan los estudiantes. En síntesis, la investigación empírica, en las últimas décadas ha demostrado que la eficacia de las escuelas para el rendimiento escolar y el logro académico de los estudiantes depende de diversas variables que no solamente dependen de la escuela, sin no del contexto sociocultural y socioeconómico de los estudiantes, siendo estas las más relacionadas al rendimiento escolar.

\section{La influencia de los padres en el rendimiento escolar}

Como hemos mencionado anteriormente, el rendimiento escolar se encuentra influenciado primordialmente por las características socioeconómica y sociocultural de los estudiantes. En este sentido, la familia representa el primer agente desarrollador de la personalidad, a través de la transmisión de actitudes, comportamientos y hábitos, de tal modo que el nivel de estudios de los padres de familia juega un papel muy importante en la generación de expectativas de los alumnos (Page, 1990). De acuerdo con (Bandura, 1987) la mayor parte de la conducta humana se aprende por observación de las conductas que otras personas 
realizan, es decir, mediante la observación de modelos. En este sentido (Romagnoli \& Cortese, 2015) han encontrado que el modelo parental de los estudiantes constituye un factor de suma trascendencia en el rendimiento escolar, debido a que las personas de la familia ejercen una gran influencia de los estudiantes hacia el estudio. Para (Davis-Kean, 2005) el nivel de estudios de los padres influye en la manera de estructurar el entorno familiar, de este modo la interacción de los padres con altos niveles de escolaridad proporciona un mayor acompañamiento y expectativas académicas a sus hijos.

En Garbanzo (2007), se le da un mayor énfasis al nivel de estudios de la madre, debido a las exigencias académicas hacia sus hijos, al delegar los padres a las madres la supervisión de la educación en el hogar. Sin embargo, en las investigaciones empíricas de (Da Cuña, Gutiérrez , Barón, \& Labajos, 2014), no han encontrado correlaciones significativas entre estudiantes con padres de familia con menor nivel de estudios y estudiantes con padres con estudios superiores. A manera de resumen, los padres con elevado nivel socioeconómico y sociocultural pueden funcionar como efectivos modelos de comportamiento y rendimiento escolar de sus hijos.

\section{Medición de gran escala del rendimiento escolar}

La mayoría de los estudios de gran escala enfatizan el uso de indicadores de logro educativo con rendimiento académico para medir la eficacia de los procesos escolares. (Backhoff, Bouzas, Contreras, Hernández, \& García, 2007). Al respecto en (Page, 1990) hace mención que las maneras más comunes para medir el rendimiento académico son las calificaciones escolares y las pruebas objetivas o también conocidas como test de rendimiento. En el caso de las calificaciones escolares, estas representan el criterio social y legal del rendimiento de un alumno, dado por una institución educativa, sin embargo, teniendo presente que las evaluaciones son realizadas según el criterio de los profesores, la información no es válida para realizar comparaciones entre centros escolares dada la subjetividad con la que se realiza las evaluaciones. De tal modo, que las pruebas objetivas como medida del rendimiento escolar, dada su rigurosidad en la validez y confianza en su elaboración, ofrecen mayores ventajas debido a que eliminan la subjetividad de los profesores y permiten realizar comparaciones entre diversos centros escolares. Sus limitaciones radican principalmente para 
valorar aspectos como la composición o la redacción, además de estimular la memorización abstracta de datos. Sin embargo, algunos estudios, que sustentan las ventajas de medir el rendimiento escolar a través de pruebas objetivas, son las de (Suárez, Elías, \& Zarza, 2016) en donde se demuestra a partir de los resultados de una prueba estandarizada, la asociación del desempeño de los estudiantes, con un conjunto de variables del contexto de los estudiantes de Paraguay.

En México, el modelo de construcción de escalas de factores asociados al aprendizaje en (Backhoff, Bouzas, Contreras, Hernández, \& García, 2007) señala como en el conjunto de seis variables: asistencia al cine, expectativas educativas de los padres (hacia sus hijos), escolaridad de la madre, escolaridad del padre, número de libros en casa y disponibilidad de Internet en el hogar. De acuerdo con las correlaciones de cada una de las variables que la componen con el aprendizaje del Español y las Matemáticas de los estudiantes de sexto de primaria, las expectativas de los padres hacia la educación de sus hijos y la escolaridad del padre y de la madre son las variables que mejor predicen el logro educativo en ambos dominios. Finalmente, si bien hay quienes critican el uso de pruebas de opción múltiple como indicadores de resultados del proceso educativo, hoy en día representan el indicador más utilizado para medir la eficacia de las escuelas

\section{Metodología}

\section{Objetivos}

Como objetivo del presente trabajo se tuvo conocer si el nivel de estudios de los padres de familia ejerce una influencia en el desempeño académico de los estudiantes de la educación profesional técnica, medido a través de una prueba de rendimiento estandarizada. De manera concreta, con el estudio tuvo los siguientes objetivos específicos:

- Determinar el nivel de estudios de las madres de los estudiantes.

- Determinar el nivel de estudios de los padres de los estudiantes.

- Establecer si existe relaciones significativas entre el nivel de estudios de las madres y el desempeño académico de estudiantes.

- Establecer si existe relaciones significativas entre el nivel de estudios de los padres y el desempeño académico de estudiantes. 


\section{Variables de estudio}

Las variables objeto de estudios son de tipo categórico y se clasificaron como a continuación se indica:

- Variable dependiente: Nivel en el desempeño de una prueba; Niveles I,II,II,IV,V y VI.

- Variable independiente: Nivel de estudios de los padres de familia: sin estudio, preescolar, primaria, secundaria, bachillerato, TSU, licenciatura, maestría, doctorado.

\section{Muestra}

La muestra contempló a un total de 10,110 estudiantes. Para su selección de la se empleó el modelo de muestreo aleatorio estratificado, teniendo presente que se cuenta con una población finita en 39 planteles. La población objetivo del estudio estuvo compuesta por estudiantes de sexto semestre (hombres y mujeres) de cada uno de los planteles del Colegio de Educación Profesional Técnica del Estado de México.

La presente indagación es tipo transeccional descriptivo de corte cuantitativo. Para dar respuesta a la pregunta de investigación se definieron las hipótesis, nula (Ho) y una hipótesis alterna (Ha): Ho. "El nivel de estudios de los padres de familia no tiene influencia en el desempeño académico de los estudiantes". Ha: "El nivel de estudios de los padres de familia tiene influencia en el desempeño académico de los estudiantes"

\section{Instrumento}

La Evaluación Institucional del Núcleo de Formación Básica del Conalep Estado de México, es un instrumento desarrollado para conocer el dominio de los aprendizajes del marco curricular común (MCC) del bachillerato, de sus planes y programas de estudio. Dicha prueba contiene un total de 57 reactivos del tipo politómico, los cuales incluyen los objetivos de aprendizaje en los cinco campos disciplinares del (MCC), como lo son las Matemáticas, Ciencias Experimentales, Ciencias Sociales, Humanidades y Lenguaje y Comunicación. De acuerdo con (Tapia, 2018) el instrumento tiene un índice de dificultad del 50\%, 56 de los 57 reactivos tienen un índice de discriminación superior a .32. Los indicadores psicométricos reflejan la calidad de dicho instrumento.

\section{Procedimiento de recolección y análisis de datos}


La recolección de datos se realizó a partir de la aplicación del instrumento en línea, en cada uno de los centros educativos mediante el uso de un formulario en Google forms ${ }^{\circledR}$. Los datos se descargaron en una hoja de cálculo en donde fueron extraídas los resultados de las variables de estudio. Posteriormente se vació los datos obtenidos en el paquete estadísticos SPSS $®$.

En el análisis de los datos se empleó un método no paramétrico para contrastar las hipótesis planteadas. En ello se hizo uso de tablas de contingencia de 8x6 a fin de identificar patrones de comportamiento de los datos obtenidos. Siguiendo este método se aplicó el contraste no paramétrico de Chi-cuadrado, el cual se basa en la comparación de frecuencias esperaras y observadas.

\section{Resultados}

Para el análisis de los resultados se emplearon técnicas de análisis de estadística descriptiva e inferencial. En las tablas 1 y 2 se muestran los resultados de la frecuencia y media del nivel de estudio de los padres de familia. En las tablas 3 y 4 se muestran las tablas de contingencia 8x6, realizada para comprobar la influencia del padre y la madre en el desempeño escolar de los estudiantes. Los datos de esta tabla, muestra la relación de la variable dependiente "nivel del desempeño de los estudiantes" (en vertical), medido a partir del establecimiento de 4 niveles o cuartiles, y la variable independiente (en horizontal) "nivel de estudios del padre", medido a partir de nueve posibilidades, según el caso.

\section{Nivel de estudios de las madres}

Tabla 1 Nivel de estudios de las madres

\begin{tabular}{|c|c|c|}
\hline $\begin{array}{c}\text { Nivel de } \\
\text { estudios }\end{array}$ & Frecuencia & $\mathrm{X}$ \\
\hline Preescolar & 29 & 0.3 \\
\hline Primaria & 2409 & 23.7 \\
\hline Secundaria & 4686 & 46 \\
\hline Bachillerato & 2230 & 21.9 \\
\hline TSU & 203 & 2 \\
\hline Licenciatura & 313 & 3.1 \\
\hline Maestría & 60 & 0.6 \\
\hline Doctorado & 9 & 0.4 \\
\hline Total & $\mathbf{1 0 1 4 4}$ & \multicolumn{1}{|c}{} \\
\cline { 1 - 2 } & &
\end{tabular}

Fuente: Elaboración propia. 
Los resultados del nivel de estudios de las madres de la tabla 1, muestran que la media de estudios se centra en la secundaria con un $46 \%$, seguido del nivel de primaria con $23.7 \%$, bachillerato con 21.9\%. De este modo, estos tres niveles de estudio de la educación obligatoria reúnen el 91.6\%. Por otra parte, la educación superior reúne el 5.1\% y estudios de posgrado el $1 \%$.

\section{Nivel de estudios de los padres}

Tabla 2 Nivel de estudios de los padres

\begin{tabular}{|c|c|c|}
\hline $\begin{array}{c}\text { Nivel de } \\
\text { estudios }\end{array}$ & Frecuencia & $\mathbf{X}$ \\
\hline Preescolar & 43 & 0.4 \\
\hline Primaria & 2059 & 20.2 \\
\hline Secundaria & 4284 & 42.1 \\
\hline Bachillerato & 2488 & 24.4 \\
\hline TSU & 270 & 2.7 \\
\hline Licenciatura & 350 & 3.4 \\
\hline Maestría & 49 & 0.5 \\
\hline Doctorado & 24 & 0.2 \\
\hline Total & 9876 & \\
Fuente: Elaboración propia. &
\end{tabular}

En el caso de los padres la tabla 2 muestra el nivel de estudios que agrupa la mayor concentración al igual que las madres es la secundaria con una media del 42.1\%, 3.9 puntos porcentuales por abajo que el de las madres, seguido del bachillerato con 24.4\%, 2.5 puntos porcentuales por arriba del resultado de las madres en este segmento y la primaria con $20.2 \%$, 3.5 puntos porcentuales por debajo de las madres de familia. Al respecto, los niveles correspondientes a la educación obligatoria los padres reúnen un total del 86.7\%, 4.9 puntos porcentuales por debajo de la media de las madres en este segmento. En cuanto a la educación superior, el $6.1 \%$ se encuentra en este segmento, $1 \%$ mayor que las madres. En el caso de posgrado corresponde el $0.7 \%, .03$ puntos menos en comparación que las madres.

\section{Influencia de los estudios de la madre en el desempeño escolar de los estudiantes}

En la tabla 3 se muestra el cruce de variables en forma $6 \times 8$, relacionando los resultados de las variables categóricas del estudio. En ella, se observa que los alumnos con madres con estudio del nivel preescolar alcanzan en mayor proporción, el nivel II de desempeño. En el caso del nivel de estudios con primaria, la mayor proporción se ubica en el nivel de desempeño III, los estudios con secundaria la mayor concentración se ubica en el nivel V, 
bachillerato en el nivel VI, TSU en el nivel IV, licenciatura en el nivel V, maestría y doctorado en el nivel II. En el caso de la prueba de contraste de variables a partir del uso de Chi-cuadrado de Pearson, se obtuvo un p valor de 73,471, con 35 grados de libertad y con una significancia asintótica de .000 . No suponiendo la hipótesis nula.

Tabla 3 Tabla de contingencia de variables (madre)

\begin{tabular}{|c|c|c|c|c|c|c|c|c|c|}
\hline & \multicolumn{6}{|c|}{ Nivel de desempeño } & \multirow{3}{*}{$\begin{array}{r}\text { Total } \\
28 \\
\end{array}$} \\
\hline & & & $\mathrm{I}$ & II & III & IV & $\mathrm{V}$ & $\mathrm{VI}$ & \\
\hline \multirow{24}{*}{ 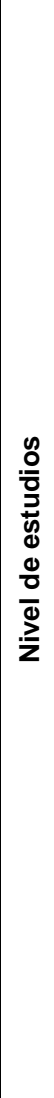 } & \multirow{3}{*}{ Preescolar } & Recuento & 0 & 9 & 12 & 4 & 2 & 1 & \\
\hline & & Recuento esperado & 0 & 6 & 12.1 & 5.9 & 2 & 2 & 28 \\
\hline & & $\begin{array}{l}\text { \% Nivel de } \\
\text { desempeño }\end{array}$ & $0.00 \%$ & $0.40 \%$ & $0.30 \%$ & $0.20 \%$ & $0.30 \%$ & $0.10 \%$ & $0.30 \%$ \\
\hline & \multirow{3}{*}{ Primaria } & Recuento & 1 & 475 & 1090 & 499 & 158 & 170 & 2393 \\
\hline & & Recuento esperado & 2.7 & 515.3 & 1032.4 & 503.5 & 171.4 & 167.7 & 2393 \\
\hline & & \begin{tabular}{|l|}
$\%$ Nivel de \\
desempeño \\
\end{tabular} & $9.10 \%$ & $22.30 \%$ & $25.60 \%$ & $24.00 \%$ & $22.30 \%$ & $24.50 \%$ & $24.20 \%$ \\
\hline & \multirow{3}{*}{ Secundaria } & Recuento & 8 & 1003 & 2037 & 945 & 352 & 315 & 4660 \\
\hline & & Recuento esperado & 5.2 & 1003.6 & 2010.4 & 980.5 & 333.7 & 326.7 & 4660 \\
\hline & & $\begin{array}{l}\text { \% Nivel de } \\
\text { desempeño }\end{array}$ & $72.70 \%$ & $47.10 \%$ & $47.80 \%$ & $45.40 \%$ & $49.70 \%$ & $45.50 \%$ & $47.10 \%$ \\
\hline & \multirow{3}{*}{ Bachillerato } & Recuento & 1 & 513 & 912 & 486 & 143 & 169 & 2224 \\
\hline & & Recuento esperado & 2.5 & 478.9 & 959.5 & 467.9 & 159.3 & 155.9 & 2224 \\
\hline & & \begin{tabular}{|l}
$\%$ Nivel de \\
desempeño
\end{tabular} & $9.10 \%$ & $24.10 \%$ & $21.40 \%$ & $23.40 \%$ & $20.20 \%$ & $24.40 \%$ & $22.50 \%$ \\
\hline & \multirow{3}{*}{ TSU } & Recuento & 0 & 45 & 73 & 56 & 10 & 16 & 200 \\
\hline & & Recuento esperado & 0.2 & 43.1 & 86.3 & 42.1 & 14.3 & 14 & 200 \\
\hline & & $\begin{array}{l}\% \text { Nivel de } \\
\text { desempeño }\end{array}$ & $0.00 \%$ & $2.10 \%$ & $1.70 \%$ & $2.70 \%$ & $1.40 \%$ & $2.30 \%$ & $2.00 \%$ \\
\hline & \multirow{3}{*}{ Licenciatura } & Recuento & 1 & 60 & 114 & 80 & 37 & 20 & 312 \\
\hline & & Recuento esperado & 0.3 & 67.2 & 134.6 & 65.6 & 22.3 & 21.9 & 312 \\
\hline & & $\begin{array}{l}\text { \% Nivel de } \\
\text { desempeño }\end{array}$ & $9.10 \%$ & $2.80 \%$ & $2.70 \%$ & $3.80 \%$ & $5.20 \%$ & $2.90 \%$ & $3.20 \%$ \\
\hline & \multirow{3}{*}{ Maestría } & Recuento & 0 & 17 & 27 & 9 & 5 & 2 & 60 \\
\hline & & Recuento esperado & 0.1 & 12.9 & 25.9 & 12.6 & 4.3 & 4.2 & 60 \\
\hline & & $\begin{array}{l}\text { \% Nivel de } \\
\text { desempeño }\end{array}$ & $0.00 \%$ & $0.80 \%$ & $0.60 \%$ & $0.40 \%$ & $0.70 \%$ & $0.30 \%$ & $0.60 \%$ \\
\hline & \multirow{3}{*}{ Doctorado } & Recuento & 0 & 7 & 0 & 1 & 1 & 0 & 9 \\
\hline & & Recuento esperado & 0 & 1.9 & 3.9 & 1.9 & 0.6 & 0.6 & 9 \\
\hline & & $\begin{array}{l}\text { \% Nivel de } \\
\text { desempeño }\end{array}$ & $0.00 \%$ & $0.30 \%$ & $0.00 \%$ & $0.00 \%$ & $0.10 \%$ & $0.00 \%$ & $0.10 \%$ \\
\hline & \multirow{3}{*}{ Total } & Recuento & 11 & 2129 & 4265 & 2080 & 708 & 693 & 9886 \\
\hline & & Recuento esperado & 11 & 2129 & 4265 & 2080 & 708 & 693 & 9886 \\
\hline & & $\begin{array}{l}\text { \% Nivel de } \\
\text { desempeño }\end{array}$ & $100 \%$ & $100 \%$ & $100 \%$ & $100 \%$ & $100 \%$ & $100 \%$ & $100 \%$ \\
\hline
\end{tabular}

Fuente: Elaboración propia.

\section{Influencia de los estudios del padre en el desempeño escolar de los estudiantes}

En la tabla 4, se observa que los alumnos con madres con estudio del nivel preescolar alcanzan en mayor proporción, el nivel IV de desempeño, el nivel de estudios con primaria y secundaria, la mayor proporción se ubicó en el nivel de desempeño I, los estudios con secundaria la mayor concentración se ubica en el nivel V, bachillerato en el nivel VI, TSU en el nivel II, licenciatura y maestría en el nivel V y doctorado en el nivel II. En el caso de la 
prueba de contraste de variables a partir del uso de Chi-cuadrado de Pearson, se obtuvo un $\mathrm{p}$ valor de 71,238, con 35 grados de libertad y con una significancia asintótica de .000 . No suponiendo la hipótesis nula.

Tabla 4 Tabla de contingencia de variables (padres)

\begin{tabular}{|c|c|c|c|c|c|c|c|c|c|}
\hline & \multicolumn{6}{|c|}{ Nivel de desempeño } & \multirow[t]{2}{*}{ Total } \\
\hline & & & I & II & II & IV & V & VI & \\
\hline \multirow{24}{*}{$\begin{array}{l}\frac{0}{0} \\
\frac{0}{2} \\
\frac{2}{0} \\
0 \\
0 \\
\frac{0}{0} \\
\frac{1}{10} \\
\frac{1}{z}\end{array}$} & \multirow{3}{*}{ Preescolar } & Recuento & 0 & 8 & 17 & 14 & 2 & 2 & 43 \\
\hline & & Recuento esperado & 0 & 9.3 & 18.5 & 9 & 3.1 & 3 & 43 \\
\hline & & \begin{tabular}{|l|}
$\%$ Nivel de \\
desempeño \\
\end{tabular} & $0.00 \%$ & $0.40 \%$ & $0.40 \%$ & $0.70 \%$ & $0.30 \%$ & $0.30 \%$ & $0.50 \%$ \\
\hline & \multirow{3}{*}{ Primaria } & Recuento & 5 & 419 & 944 & 407 & 151 & 122 & 2048 \\
\hline & & Recuento esperado & 2.4 & 443.3 & 881.2 & 430.2 & 146.5 & 144.4 & 2048 \\
\hline & & $\begin{array}{l}\% \text { Nivel de } \\
\text { desempeño }\end{array}$ & $45.50 \%$ & $20.30 \%$ & $23.10 \%$ & $20.40 \%$ & $22.20 \%$ & $18.20 \%$ & $21.50 \%$ \\
\hline & \multirow{3}{*}{ Secundaria } & Recuento & 5 & 916 & 1847 & 886 & 304 & 299 & 4257 \\
\hline & & Recuento esperado & 4.9 & 921.4 & 1831.7 & 894.2 & 304.6 & 300.1 & 4257 \\
\hline & & $\begin{array}{l}\% \text { Nivel de } \\
\text { desempeño }\end{array}$ & $45.50 \%$ & $44.50 \%$ & $45.10 \%$ & $44.30 \%$ & $44.60 \%$ & $44.60 \%$ & $44.70 \%$ \\
\hline & \multirow{3}{*}{ Bachillerato } & Recuento & 1 & 555 & 1040 & 534 & 163 & 185 & 2478 \\
\hline & & Recuento esperado & 2.9 & 536.4 & 1066.2 & 520.5 & 177.3 & 174.7 & 2478 \\
\hline & & $\begin{array}{l}\text { \% Nivel de } \\
\text { desempeño }\end{array}$ & $9.10 \%$ & $26.90 \%$ & $25.40 \%$ & $26.70 \%$ & $23.90 \%$ & $27.60 \%$ & $26.00 \%$ \\
\hline & \multirow{3}{*}{ TSU } & Recuento & 0 & 75 & 106 & 50 & 18 & 21 & 270 \\
\hline & & Recuento esperado & 0.3 & 58.4 & 116.2 & 56.7 & 19.3 & 19 & 270 \\
\hline & & $\begin{array}{l}\% \text { Nivel de } \\
\text { desempeño }\end{array}$ & $0.00 \%$ & $3.60 \%$ & $2.60 \%$ & $2.50 \%$ & $2.60 \%$ & $3.10 \%$ & $2.80 \%$ \\
\hline & \multirow{3}{*}{ Licenciatura } & Recuento & 0 & 67 & 123 & 90 & 36 & 32 & 348 \\
\hline & & Recuento esperado & 0.4 & 75.3 & 149.7 & 73.1 & 24.9 & 24.5 & 348 \\
\hline & & $\begin{array}{l}\text { \% Nivel de } \\
\text { desempeño }\end{array}$ & $0.00 \%$ & $3.30 \%$ & $3.00 \%$ & $4.50 \%$ & $5.30 \%$ & $4.80 \%$ & $3.70 \%$ \\
\hline & \multirow{3}{*}{ Maestría } & Recuento & 0 & 8 & 16 & 12 & 6 & 7 & 49 \\
\hline & & Recuento esperado & 0.1 & 10.6 & 21.1 & 10.3 & 3.5 & 3.5 & 49 \\
\hline & & $\begin{array}{l}\% \text { Nivel de } \\
\text { desempeño }\end{array}$ & $0.00 \%$ & $0.40 \%$ & $0.40 \%$ & $0.60 \%$ & $0.90 \%$ & $1.00 \%$ & $0.50 \%$ \\
\hline & \multirow{3}{*}{ Doctorado } & Recuento & 0 & 12 & 2 & 6 & 1 & 3 & 24 \\
\hline & & Recuento esperado & 0 & 5.2 & 10.3 & 5 & 1.7 & 1.7 & 24 \\
\hline & & \begin{tabular}{|l|}
$\%$ Nivel de \\
desempeño \\
\end{tabular} & $0.00 \%$ & $0.60 \%$ & $0.00 \%$ & $0.30 \%$ & $0.10 \%$ & $0.40 \%$ & $0.30 \%$ \\
\hline & \multirow{3}{*}{ Total } & Recuento & 11 & 2060 & 4095 & 1999 & 681 & 671 & 9517 \\
\hline & & Recuento esperado & 11 & 2060 & 4095 & 1999 & 681 & 671 & 9517 \\
\hline & & $\begin{array}{l}\text { \% Nivel de } \\
\text { desempeño }\end{array}$ & $100 \%$ & $100 \%$ & $100 \%$ & $100 \%$ & $100 \%$ & $100 \%$ & $100 \%$ \\
\hline
\end{tabular}

Fuente: Elaboración propia.

\section{Conclusiones}

En la presente investigación se realizó un análisis cuantitativo de la influencia del nivel de estudios de los padres en el desempeño de 10,110 estudiantes se sexto semestre del Conalep Estado de México, a partir de la aplicación de una prueba estandarizada de evaluación del logro de los aprendizajes. 
En atención a los objetivos de la investigación se determinó que el promedio máximo de estudios de los padres de familia es la educación secundaria, ocupando en promedio poco menos del 50\% de la totalidad. En respuesta a la pregunta de investigación, de determinó a partir de la hipótesis de investigación que existe una influencia de la variable "Nivel de estudios de los padres de familia y el nivel de desempeño de los estudiantes", en el análisis realizado a partir del método estadístico de Chi Cuadrado para modelos no paramétricos se obtuvo que la influencia del nivel de estudios de las madres de familia es más fuerte que el de los padres de familia corroborando de este modo los resultados de (Garbanzo, 2007) en donde el nivel de estudios de la madre, genera mayor influencia en el desempeño de los estudiantes, al delegar los padres a las madres la supervisión de la educación en el hogar.

\section{Referencias}

Backhoff, E., Bouzas, A., Contreras, A., Hernández, E., \& García, M. (2007). Factores escolares y de aprendizaje en México: El caso de la educación básica. México: Instituto Nacional para la Evaluación Educativa.

Backhoff, E., Monroy, L., \& Tanamachi, M. (2005). Cuestionarios de Contexto de las pruebas Excale: Marco de referencia. México: Instituto Nacional para la Evaluación de la Educación (INEE). .

Bandura, A. (1987). Teoría del aprendizaje social. Madrid: Espasa-Calpe.

Coleman, J., Campbell, E., Hobson, C., McPartland, J., Mood, A., Weinfeld, F., \& York, R. (1966). Equqlity of educational opportunity. Washington: Government Printing Office.

Da Cuña, I., Gutiérrez , M., Barón, F., \& Labajos, M. (2014). Influencia del nivel educativo de los padres en el rendimiento académico, las estrategias de aprendizaje y estilos de aprendizaje desde la perspectiva de género . Revista de estilos de aprendizaje, 64-84.

Davis-Kean. (2005). The influence of parent education and family income on child achievement: the indirect role of parental expectations and the home environment. . Journal of Family Psychology, 294-304. 
Garbanzo, G. (2007). Factores asociados al rendimiento académico en estudiantes universitarios, una reflexión desde la calidad de la educación superior pública. Revista Educación, 43-63.

INEE. (2015). Informe PLANEA. México: Instituto para la Evaluación Educativa.

INEE. (2017). PLANEA Resultados Nacionales 2017. Obtenido de http://planea.sep.gob.mx/content/general/docs/2017/ResultadosNacionalesPlaneaM S2017.PDF

Jiménez, M. (2000). Competencia social: intervención preventiva en la escuela. Infancia y Sociedad, 21-48.

Martínez-Otero, V. (2007). Los adolescentes ante el estudio. Causas y consecuencias del rendimiento académico. Madrid: Fundamentos.

Morales, L., Morales, V., \& Holguín, S. (2016). Rendimiento escolar. Humanidades, Tecnología y Coiencia, del Instituto Politécnico Nacional, 1-5.

Page, A. (1990). Hacia un modelo causal en el rendimiento académico. Madrid: Centro del Ministerio de Educacón y Ciencia: C.I.D.E.

Romagnoli , C., \& Cortese, I. (2015). ¿Cómo la familia influye en el aprendizaje y rendimiento escolar? . Santiago: Disponible en Centro de Recursos VALORAS: www.valoras.uc.cl.

SEP. (15 de septiembre de 2017). gob.mx. Obtenido de PLANEA, SEP: http://planea.sep.gob.mx/ms/\#aplica_ms

Suárez, S., Elías, R., \& Zarza, D. (2016). Factores Asociados al Rendimiento Académico de Estudiantes de Paraguay: Un Análisis de los Resultados del TERCE. REICE. Revista Iberoamericana sobre Calidad, Eficacia y Cambio en Educación, 113-133. 
Tapia, I. (2018). Metodología para la Evaluación de los aprendizajes del marco curricular comú del bachillerato. Revista Congreso Mesoamericano de Investigación UNACH, 540-544. 